\title{
Diffusion Kurtosis MR Imaging versus Conventional Diffusion-Weighted Imaging for Distinguishing Hepatocellular Carcinoma from Benign Hepatic Nodules
}

\author{
Yingmei Jia, ${ }^{1}$ Huasong Cai, ${ }^{1}$ Meng Wang, ${ }^{1}$ Yanji Luo, ${ }^{1}$ Ling Xu, ${ }^{2}$ Zhi Dong, ${ }^{1}$ Xu Yan, ${ }^{3}$ \\ Zi-Ping Li $\mathbb{1}{ }^{1},{ }^{1}$ and Shi-Ting Feng $\mathbb{1}^{1}$ \\ ${ }^{1}$ Department of Radiology, The First Affiliated Hospital, Sun Yat-Sen University, 58th, The Second Zhongshan Road, Guangzhou, \\ Guangdong 510080, China \\ ${ }^{2}$ Faculty of Medicine and Dentistry, University of Western Australia, Perth, Australia \\ ${ }^{3}$ MR Collaboration NE Asia, Siemens Healthcare, Shanghai, China
}

Correspondence should be addressed to Zi-Ping Li; liziping163@163.com and Shi-Ting Feng; fengsht@mail.sysu.edu.cn

Received 25 January 2019; Revised 9 June 2019; Accepted 3 July 2019; Published 17 July 2019

Academic Editor: Luca Filippi

Copyright $\odot 2019$ Yingmei Jia et al. This is an open access article distributed under the Creative Commons Attribution License, which permits unrestricted use, distribution, and reproduction in any medium, provided the original work is properly cited.

Objectives. To assess the efficacy of diffusion kurtosis imaging (DKI) and compare DKI-derived parameters with conventional diffusion-weighted imaging (DWI) for distinguishing hepatocellular carcinoma (HCC) from benign hepatic nodules including focal nodular hyperplasia (FNH), hemangioma, and hepatocellular adenoma (HCA). Materials and Methods. 151 patients with 182 hepatic nodules (114 HCCs and 68 benign nodules including 33 FNHs, 29 hemangiomas, and 6 HCAs) were analyzed. Preoperative MRI examinations including DKI ( $b$ values: $0,200,500,800,1500$, and $2000 \mathrm{sec} / \mathrm{mm}^{2}$ ) were performed, and kurtosis $(K)$, diffusivity $(D)$, and apparent diffusion coefficient (ADC) were calculated. The efficacy of DKI-derived parameters $K, D$, and ADC for distinguishing HCC from these benign nodules was analyzed. Results. ROC (receiver operating characteristic curve) analysis showed the optimal cutoff values of ADC, $D$, and $K$ for identification of these benign nodules, and HCCs were 1.295 (area under the curve (AUC): 0.826; sensitivity $80.6 \%$; specificity $70.8 \%$ ), 1.787 (AUC: 0.770 ; sensitivity $83.6 \%$; specificity $59.6 \%$ ), and 1.002 (AUC: 0.761 ; sensitivity 65.5\%; specificity 79.0\%), respectively. Statistically significant differences were found in ADC, $D$, and $K$ values between groups of HCC-FNH and HCC-hemangioma $(P<0.05)$. There were significant differences in $K$ and ADC values between groups of FNH-hemangioma and HCA-hemangioma $(P<0.05)$, respectively. Using logistic regression analysis, a regression equation was obtained: $\operatorname{Logit}(P)=-1.982 X_{1}+1.385 X_{3}+1.948\left(X_{1}: \mathrm{ADC} ; X_{3}: K\right)$, and odds ratios $(\mathrm{OR})$ were 0.138 (95\% confidence interval (CI): 0.052, 0.367), and 8.996 (95\% CI: 0.970, 16.460), respectively. Conclusion. Both ADC value and DKI-derived parameters $K$ and $D$ values have demonstrated a higher preoperative efficacy in distinguishing HCC from FNH, hemangioma, and HCA. No evidence was shown to suggest $D$ or $K$ value was superior to the ADC value.

\section{Introduction}

Liver nodules are commonly encountered on clinical investigations with a wide differential diagnosis. With the rapid development of imaging modalities and the continuous improvement of their sensitivity, the incidence of liver nodules detection has increased in the recent decades [1]. Liver nodules can be classified according to neoplastic nature of the lesions, including benign or malignant neoplasms as well as tumor-like lesions [2]. Hepatocellular carcinoma
(HCC) is the most common cause and is responsible for approximately $90 \%$ of primary malignancies in the liver [3-5]. Accurate preoperative diagnosis and identification of HCC are essential in determining the optimal treatment. More and more newer imaging techniques have been developed to improve the preoperative diagnostic accuracy of HCC [6].

Magnetic resonance imaging (MRI) is an important technique used for risk stratification and treatment planning in patients with HCC. Dynamic contrast-enhanced imaging 
is the most commonly used MRI sequence for identification of benign and malignant liver nodules. However, some HCCs may exhibit similar appearances to other benign hepatic nodules, such as focal nodular hyperplasia (FNH), hemangioma, and hepatocellular adenoma (HCA) [7]. These overlapping radiological characteristics result in variable specificity in HCC detection [8]. The combination of hypointensity on T1-weighted images, hyperintensity on T2weighted images, and arterial phase enhancement with washout in the portal venous on dynamic gadolinium-enhanced images may be the most common appearance of HCC on MR imaging. However, atypical radiological features may confuse the diagnosis of HCC with other benign hepatic nodules. Small HCCs less than or equal to $15 \mathrm{~mm}$ are frequently isointense on both $\mathrm{T} 1$ - and T2-weighted images and do not demonstrate the typical contrast enhancement during arterial phase images because of its hypovascular structure [9]. Furthermore, the use of gadolinium can be limited in patients with chronic renal failure due to the risk of nephrogenic systemic fibrosis, resulting in a more difficult radiological diagnosis of HCC in this group of patients [10]. Radiological differentiation of HCC from other benign hepatic nodules remains challenging.

As one of the additional nonenhanced MRI methods, diffusion-weighted imaging (DWI) has been proposed to improve the diagnostic specificity of DCE imaging for liver nodules. DWI was demonstrated to be a useful adjunct sequence to DCE imaging, improving diagnostic accuracy of liver nodules compared to DCE imaging alone. Apparent diffusion coefficient (ADC) is obtained by the monoexponential model of conventional DWI with the assumption that water diffusion obeys the Gaussian law [11]. Previous studies have reported DWI has improved the sensitivity and detection rate of HCC when combined with enhanced MRI [12]. However, some reports have also suggested that it may be difficult to differentiate HCC from other solid hepatic lesions like FNH, HCA, and metastasis by DWI due to the overlapping ADC values between HCC and these solid lesions as a result of their increased cellularity $[11,13,14]$. In 2005, Diffusion kurtosis MR imaging (DKI), first proposed by Jensen et al. [15], was described as water diffusion deviates from the Gaussian law due to the complexity of microenvironment, such as cell membranes, vessels, or macromolecules. DKI can provide information about the heterogeneity and irregularity of tissue components. Compared with the ADC value of conventional DWI, the parameters of DKI may have greater potential in reflecting the characteristics of the HCC microstructure [16-18].

The role of applying DKI to improve lesion detection and disease grading has been previously investigated in neurological, renal, prostate, and breast malignancies [19-22]. In terms of its use in HCC detection, Wang et al. [23] showed that higher mean kurtosis values are potential predictors for microvascular invasion (MVI) which is indicative of HCC. Budjan et al. [24] reported that DKI was feasible and enables quantitative differentiation between malignant and benign liver lesions. However, whether DKI is superior to DWI in distinguishing $\mathrm{HCC}$ from other liver nodules remains unclear. Thus, in this retrospective study, we aimed to explore the application value of DKI with higher $b$ values in differentiating hepatic nodules by calculating and analyzing the correlations between various parameters of conventional DWI and DKI in different hepatic lesions, respectively.

\section{Materials and Methods}

2.1. Subjects. The study was approved by the local institute review board. The requirement for informed consent was waived off.

Our study retrospectively collected 238 patients at the First Affiliated Hospital, Sun Yat-Sen University, between March 2013 and November 2016. All patients underwent MRI before the treatment was commenced. 87 patients were excluded in accordance with the following exclusion criteria: (1) patients who have been commenced on any treatment prior to MRI examination (31 cases); (2) patients without DKI sequence performed during their MRI examination (35 cases); (3) patients with lesions less than $10 \mathrm{~mm}$ in diameter only (8 cases); (4) patients whose images had severe motion artifacts (13 cases). In the end, 151 patients (119 males and 32 females, with median age of 50 years) were included in the study with 182 hepatic nodules (114 HCC and 68 benign nodules including $33 \mathrm{FNHs}$, 29 hemangioma, and 6 HCAs) (Figure 1).

All of the HCCs and HCAs were confirmed by surgery or biopsy. FNH and hemangioma were diagnosed on the basis of typical MR imaging features. FNH was diagnosed when all of the following findings were identified in the lesion: (1) isointense or slightly hypointense compared with the hepatic parenchyma on T1-weighted images (T1WI). (2) Isointense or slightly hyperintense on T2-weighted images (T2WI). (3) Homogeneous enhancement during the hepatic arterial phase. (4) Isointense or slightly hyperintense in relation to the adjacent liver parenchyma during hepatic venous and delayed phases. (5) A visible central scar seen as a hyperintense focus on T2WI and as hypointense on unenhanced T1WI, with GdEOB-DTPA uptake during the hepatobiliary phase (HBP) [25]. Hemangioma was diagnosed when the typical radiological findings were identified in the lesions, such as high signal intense compared with the liver on T2WI, peripheral globular enhancement, early total enhancement, presence of the fill in the phenomenon, and prolonged enhancement during the equilibrium phase [26].

\subsection{MRI Protocol}

2.2.1. Routine MRI Scan of the Liver. All MRI examinations were performed using a clinical $3 \mathrm{~T}$ whole-body system (Magnetom Verio, Siemens Healthcare Sector, Erlangen, Germany) with body array coil (3T; 8-channel body matrix coil). All patients were fasted for $4 \sim 6$ hours prior to examination and received training regarding breath holding. All images were obtained in supine position.

MRI sequences: Fast low-angle shot (FLASH) T1WI in/ out of phase sequence axial imaging; T1WI FLASH with fat suppression (FS) sequence axial imaging; half-Fourier single-shot turbo spin-echo (HASTE) T2WI sequence axial imaging; axial and coronal postcontrast multiphase three- 


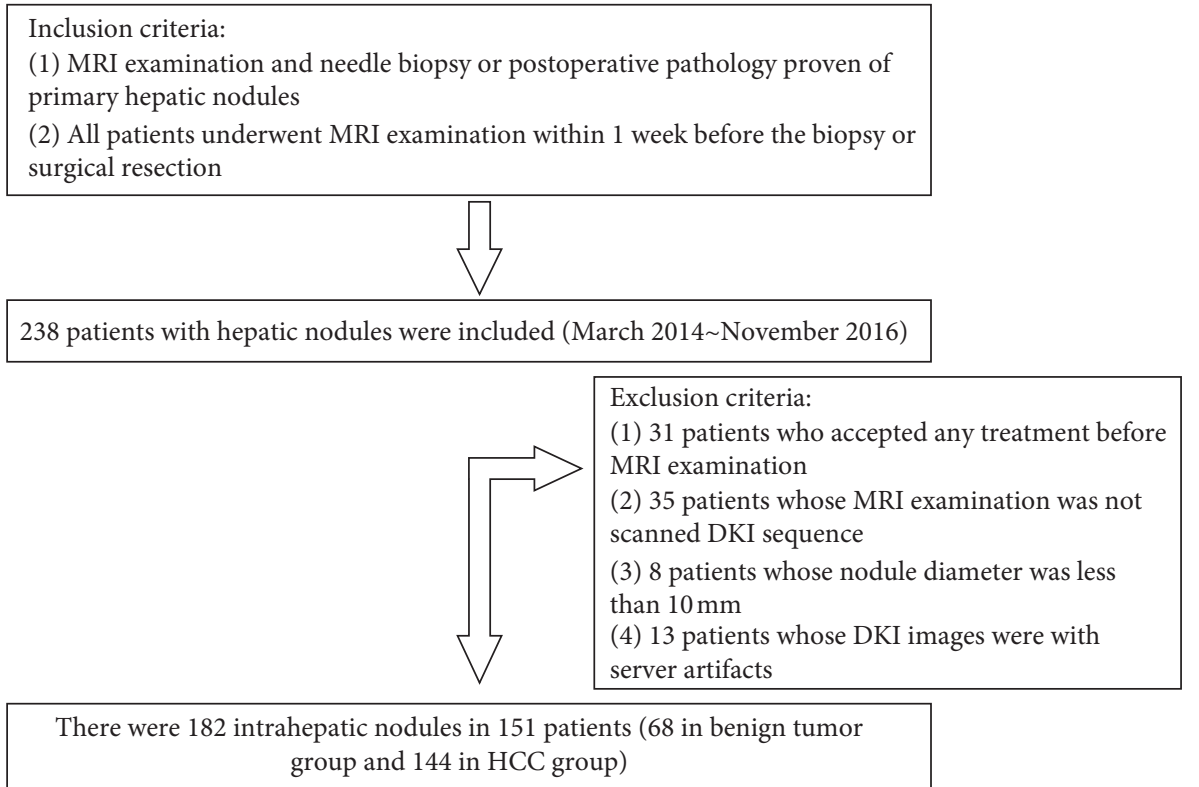

Figure 1: Flowchart of the selection process.

dimensional volume interpolated breath-hold test (3D VIBE) T1WI (FS) sequence (Table 1).

Dynamic contrast-enhanced MR imaging was performed with Gd-EOB-DTPA (Primovist ${ }^{\circledR}, 0.1 \mathrm{ml} / \mathrm{kg}$ body weight) administered as bolus injection with a flow rate of $1 \mathrm{ml} / \mathrm{s}$. Arterial phase, portal phase, and equilibrium phase images were obtained by performing 3D VIBE T1WI (FS) sequence during suspended respiration at $30-35$ seconds, 65-70 seconds, and 100-120 seconds after injection, respectively. Additional HBP images were obtained at 20 minutes after injection.

2.2.2. DKI Sequence. DKI sequences were acquired 10 minutes consistently after contrast media administration. The DKI sequence was scanned by axial freebreathing interleaved multislice DWI after contrast agent administration [27], using a single-shot spin-echo echo planar imaging (SE-EPI) sequence with $b$ values of 0,200 , $500,800,1500$, and $2000 \mathrm{~s} / \mathrm{mm}^{2}$ in three orthogonal directions. Fat suppression was achieved by inversion recovery (Table 1).

2.2.3. Image Analysis. Diffusion-weighted imaging and DKI data were analyzed by using in-house prototype software and on the basis of Matlab (MathWorks, Natick, MA). For the DKI model, the multiple b-value data $\left(0-2000 \mathrm{~s} / \mathrm{mm}^{2}\right)$ were fitted to the following equation: $S(b)=S_{0}$. $\exp \left(-b \cdot D+b^{2} \cdot D^{2} \cdot K / 6\right)$, in which $S($ b) represents the signal intensity in arbitrary units and $b$ represents the $b$ value $\left(\mathrm{s} / \mathrm{mm}^{2}\right)$, exp represents exponential function, D represents a corrected ADC accounting for non-Gaussian behavior $\left(10^{-3} \mathrm{~mm}^{2} / \mathrm{s}\right)$, and $\mathrm{K}$ represents the apparent kurtosis coefficient.

The ADC map was generated from the same data set $(b=0, b=800)$ based on the monoexponential model
$S(b)=S_{0} \cdot \exp (-b \cdot \mathrm{ADC})$ by using in-house prototype software (MathWorks, Natick, MA).

All three parameters were independently measured by two experienced gastrointestinal radiologists who were blinded to patients' information. ROIs (regions of interest) were drawn with the same criteria on the level of maximum transactional diameter of the lesion manually, avoiding the obvious areas of hemorrhage, necrosis, and cystic changes (Figures 2-5).

2.3. Statistical Analysis. All data were analyzed using the SPSS 20.0 software. Numerical data were expressed as mean $\pm \mathrm{SD}$. The Chi-squared test and one-way analysis of variance (ANOVA) were used to compare gender and age distribution amongst the HCC and hepatic benign nodules groups. The independent sample $t$-test or $K$ nonparametric test was performed for analysis of ADC, $D$, and $K$ between groups of HCC, FNH, and hemangioma.

The ROC (receiver operating characteristic curve) analysis and logistic regression method were used to compare the difference of diagnostic efficacy amongst the parameters. $P$ values $<0.05$ were considered statistically significant.

\section{Results}

182 cases of liver nodules were collected, including 68 cases of hepatic benign nodules group and 144 cases of HCC group. Gender and age distribution of all groups are listed in Table 2. Statistically significant differences in both gender and age were observed amongst the groups $(P<0.001)$.

At the test level of $\alpha=0.10$, distribution of the parameters of ADC, $D$, and $K$ was skewed, so the nonparametric test was chosen. Using the Mann-Whitney $U$ test, statistically 
TABLE 1: Acquisition parameters of the MRI protocol.

\begin{tabular}{|c|c|c|c|c|c|c|c|c|c|}
\hline Sequence & $\begin{array}{l}\text { Imaging } \\
\text { direction }\end{array}$ & Category & $\begin{array}{c}\mathrm{TR} \\
(\mathrm{ms}) \\
\end{array}$ & $\begin{array}{l}\mathrm{TE} \\
(\mathrm{ms})\end{array}$ & $\begin{array}{l}\text { FOV } \\
(\mathrm{mm})\end{array}$ & Matrix & FA $\left({ }^{\circ}\right)$ & Bandwidth & $\begin{array}{l}\text { Slice thickness } \\
(\mathrm{mm})\end{array}$ \\
\hline T1WI in-phase/out-phase & Axial & FLASH & 200 & $2.2 / 3.7$ & $328 \times 350$ & $192 \times 256$ & 65 & $930 / 977$ & 6 \\
\hline $\begin{array}{l}\text { T1WI FS } \\
\text { Contrast-enhanced }\end{array}$ & Axial & FLASH & 235 & 2.2 & $328 \times 350$ & $240 \times 320$ & 70 & 822 & 6 \\
\hline T1WI & Axial & VIBE & 3.3 & 1.2 & $328 \times 350$ & $128 \times 256$ & 13 & 501 & 2 \\
\hline T2WI & Axial & FSE & 2000 & 95.0 & $328 \times 350$ & $204 \times 256$ & 150 & 781 & 6 \\
\hline T2WI FS & Axial & FSE & 2000 & 95.0 & $328 \times 350$ & $204 \times 256$ & 150 & 781 & 6 \\
\hline DKI & Axial & EPI & 4500 & 66 & $380 \times 380$ & $128 \times 128$ & 90 & 1954 & 6 \\
\hline HBP T1WI & Axial & VIBE & 3.3 & 1.2 & $328 \times 350$ & $154 \times 256$ & 35 & 501 & 2 \\
\hline
\end{tabular}

MRI, magnetic resonance imaging; TR, repetition time; TE, echo time; FOV, field of view; T1WI, T1-weighted imaging; T2WI, T2-weighted imaging; FS, fat saturation; DKI, diffusion kurtosis imaging; HBP, hepatobiliary phase; FA, flip angle; FLASH, fast low-angle shot; VIBE, volume interpolated breath-hold examination; HASTE, half-Fourier single-shot turbo spin-echo; EPI, echo planar imaging; FSE, fast spin echo.
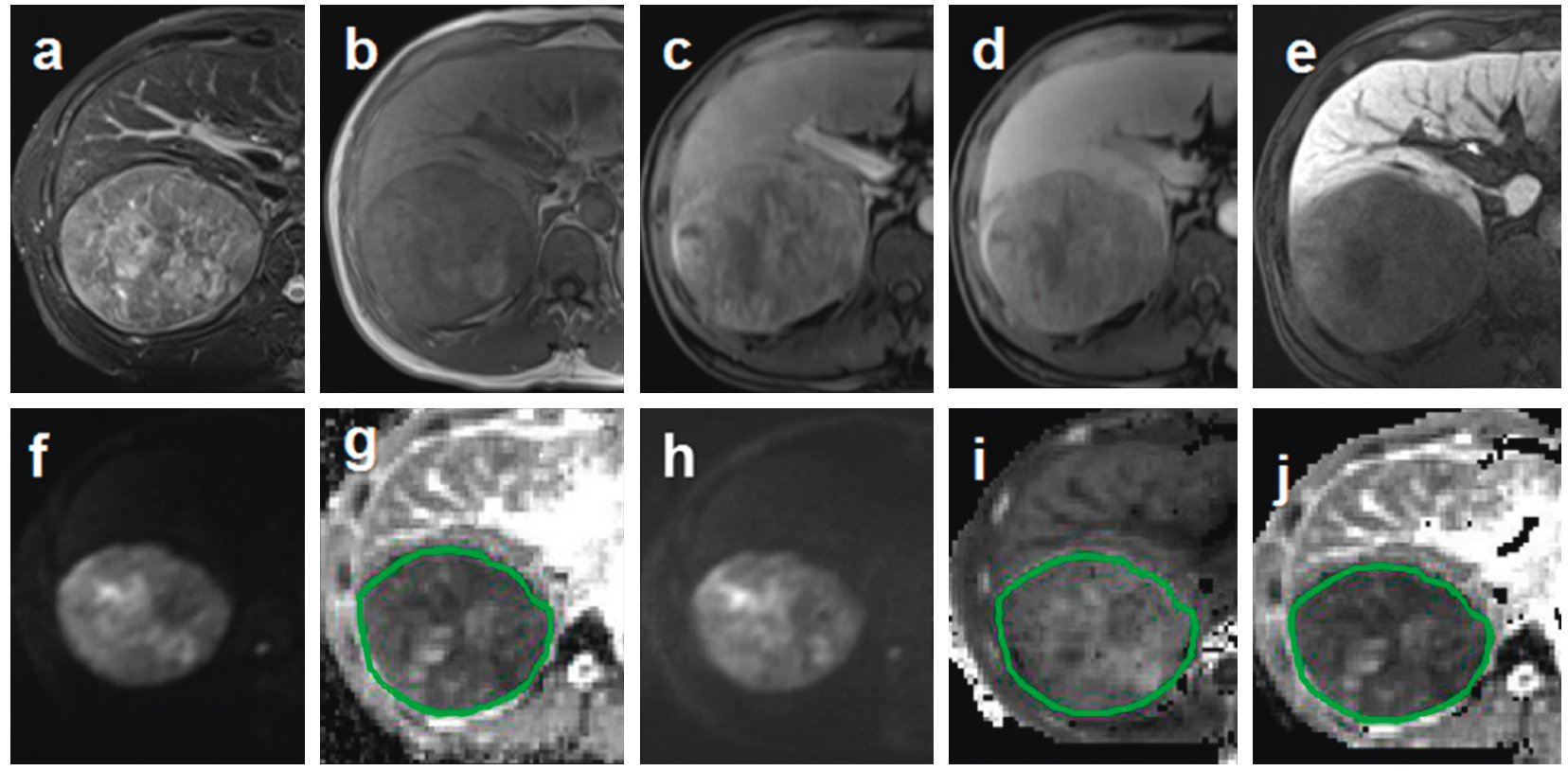

Figure 2: ROI was drawn on the level of maximum transactional diameter of HCC (green circle). (a, b) Axial fat suppress T2WI and T1WI. (c-e) Dynamic enhancement imaging including artery phase, portal phase, and hepatobiliary phase (HBP). (f, g) Axial liver DWI $\left(b=800 \mathrm{~s} / \mathrm{mm}^{2}\right)$ and ADC maps. (h-j) Axial liver DKI $\left(b=2000 \mathrm{~s} / \mathrm{mm}^{2}\right)$ and DKI-derived parameters $K$ and $D$ maps.

significant difference with respect to ADC, $D$, and $K$ was observed between the groups (Table 3). Furthermore, statistically significant difference with respect to $\mathrm{ADC}, D$, and $K$ was observed between HCC-to-FNH and HCC-to-hemangioma $(P<0.05)$. A significant difference was observed with respect to $K$ between $\mathrm{FNH}$ and hemangioma groups $(P=0.001)$. There was no significant difference in the spectrum of ADC and $D$ between FNH and hemangioma groups $(P>0.05)$. The $P$ values of parameters in 3 groups are shown in Table 4.

On ROC analysis, the optimal cutoff values of ADC, $D$, and $K$ for differentiation of HCC from other benign hepatic nodules were 1.295 (area under the curve (AUC): 0.826; sensitivity: $80.6 \%$; specificity: $70.8 \%$ ), 1.787 (AUC: 0.770; sensitivity: $83.6 \%$; specificity: $59.6 \%$ ), and 1.002 (AUC: 0.761 ; sensitivity: 65.5\%; specificity: $79.0 \%$ ), respectively (Figure 6).
Using single factor of the logistic analysis, on the test level of $P<0.1$, there was statistically significant difference in ADC, $D$, and $K$ values $(P<0.001)$, where $X_{1}, X_{2}$, and $X_{3}$ are ADC, $D$, and $K$, respectively. Afterwards, multivariate logistic regression analysis was used to obtain the regression equation with the forward stepwise method: $\operatorname{Logit}(P)=-1.982 X_{1}+1.385 X_{3}+1.948, \quad P<0.001, \quad 0.55$, and odds ratio values were 0.138 ( $95 \%$ confidential interval: $0.052,0.367)$ and 8.996 (95\% confidential interval: 0.970 , 16.460), respectively. Above all, ADC can be used as a separate protective factor, while the $D$ and $K$ values cannot be used as a single risk factor.

\section{Discussion}

Our study demonstrated that both ADC and DKI-derived parameters $K$ and $D$ values can be used to distinguish HCC 

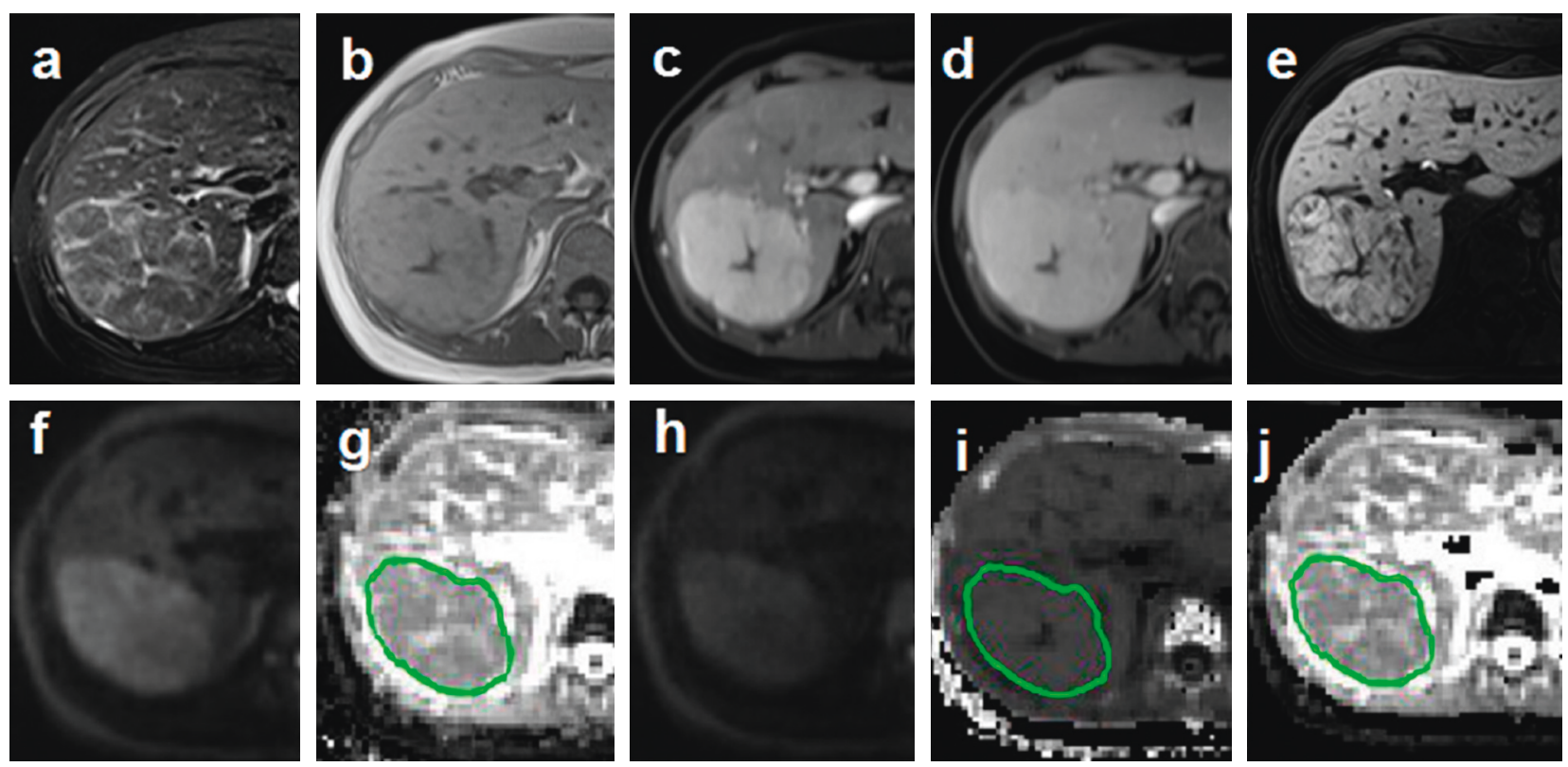

Figure 3: ROI was drawn on the level of maximum transactional diameter of FNH (green circle). (a, b) Axial fat suppress T2WI and T1WI. (c-e) Dynamic enhancement imaging included artery phase, portal phase, and hepatobiliary phase (HBP). (f, g) Axial liver DWI $\left(b=800 \mathrm{~s} / \mathrm{mm}^{2}\right)$ and ADC maps. (h-j) Axial liver DKI $\left(b=2000 \mathrm{~s} / \mathrm{mm}^{2}\right)$ and DKI-derived parameters $K$ and $D$ maps.
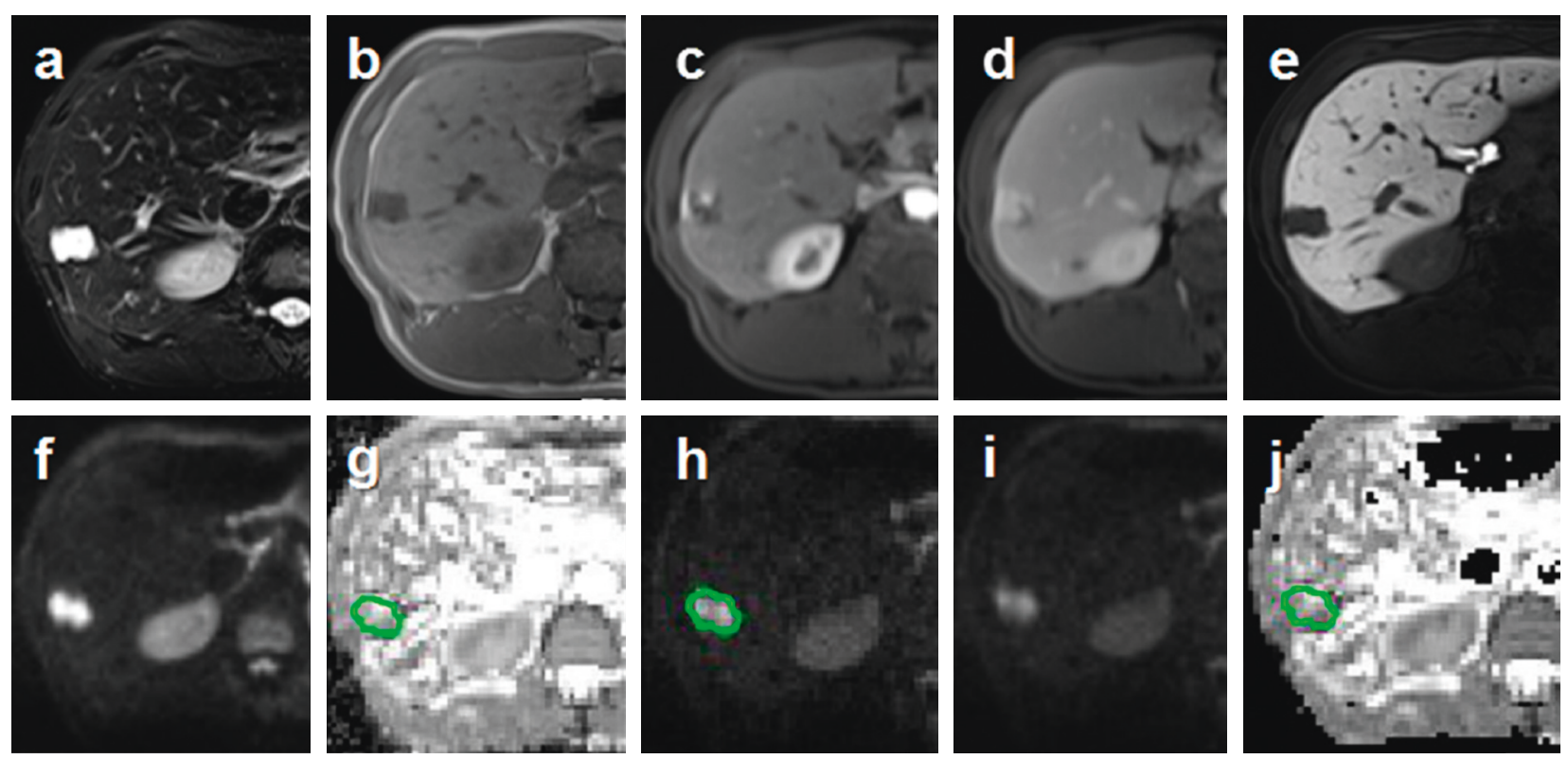

FIgURE 4: ROI was drawn on the level of maximum transactional diameter of hemangioma (green circle). (a, b) Axial fat suppress T2WI and T1WI. (c-e) Dynamic enhancement imaging included artery phase, portal phase, and hepatobiliary phase (HBP). (f, g) Axial liver DWI $\left(b=800 \mathrm{~s} / \mathrm{mm}^{2}\right)$ and ADC maps. (h-j) Axial liver DKI $\left(b=2000 \mathrm{~s} / \mathrm{mm}^{2}\right)$ and DKI-derived parameters $K$ and $D$ maps.

from those benign hepatic nodules such as FNH, hemangioma, and HCA. The sensitivity of the $D$ value was higher than those of ADC and $K$ values. We also found that FNH and hemangioma could be distinguished from HCC by using ADC, $D$, and $K$ values. However, only the $K$ value could differentiate $\mathrm{FNH}$ from hemangioma, and the ADC value could differentiate HCA from hemangioma.

As a form of MR imaging, DWI bases on measuring the random Brownian motion of water molecules within a voxel of tissue. Highly cellular tissues or those with cellular swelling exhibit lower diffusion coefficients. As an MRI, it is well known that DWI has been proved to be highly sensitive in detection of HCC. However, in particular when interpreted in combination with enhanced MR, the difference of ADC between the benign liver nodules and HCC remains controversial in the previous studies [28]. Some previous studies have showed that benign lesions had higher ADC values than those of malignant lesions, with a variable degree of overlap. 

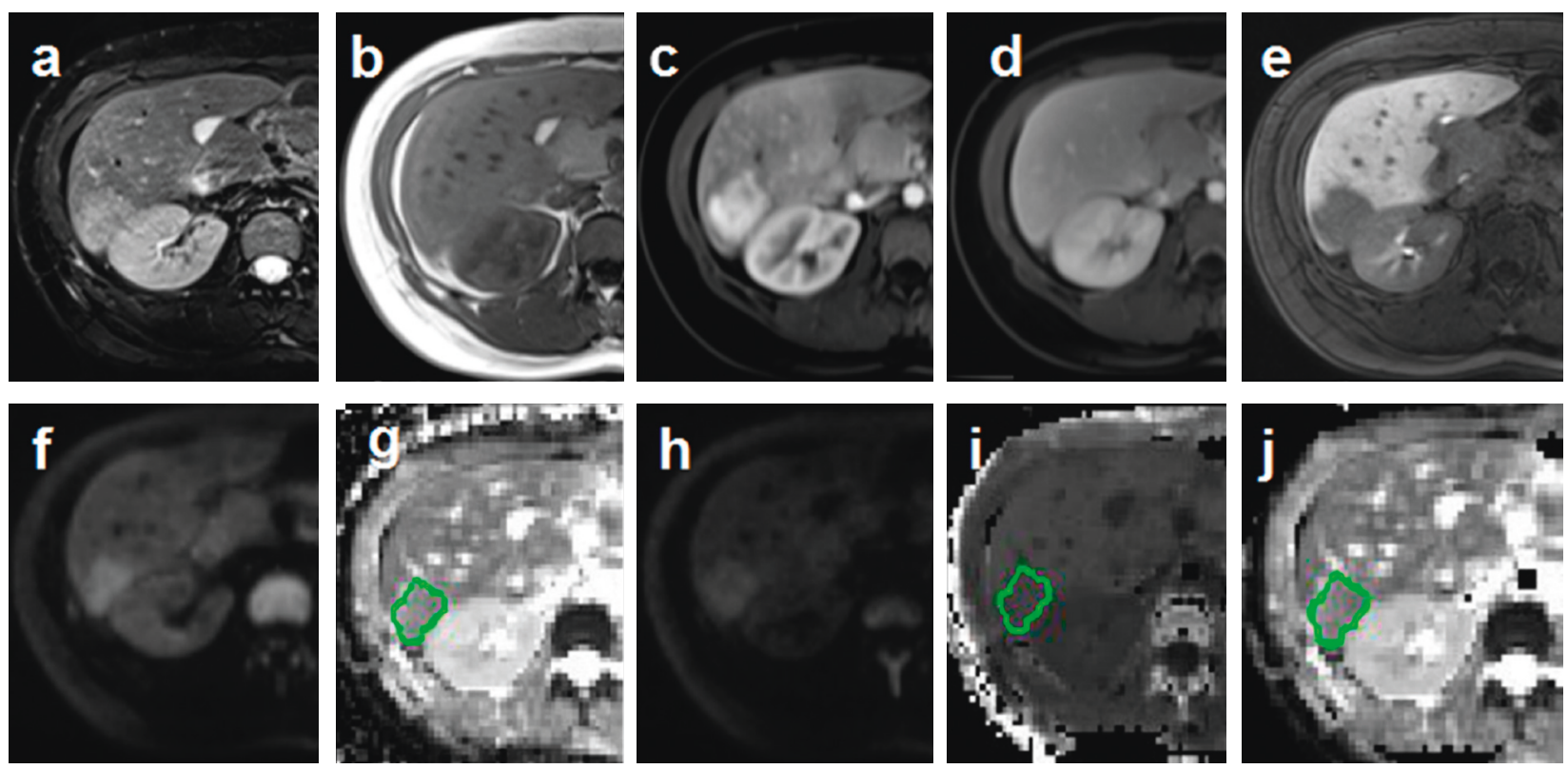

Figure 5: ROI was drawn on the level of maximum transactional diameter of HCA (green circle). (a, b) Axial fat suppress T2WI and T1WI. (c-e) Dynamic enhancement imaging included artery phase, portal phase, and hepatobiliary phase (HBP). (f, g) Axial liver DWI $\left(b=800 \mathrm{~s} / \mathrm{mm}^{2}\right)$ and ADC maps. (h-j) Axial liver DKI $\left(b=2000 \mathrm{~s} / \mathrm{mm}^{2}\right)$ and DKI-derived parameters $K$ and $D$ maps.

TABle 2: Comparison of gender and age distribution amongst HCC, FNH, and HCA groups.

\begin{tabular}{|c|c|c|c|c|c|}
\hline & $\mathrm{FNH}$ & Hemangioma & $\mathrm{HCA}$ & $\mathrm{HCC}$ & $P$ value \\
\hline Age, yrs, mean $\pm S D$ & $37.0 \pm 1.8$ & $42.5 \pm 2.5$ & $41.0 \pm 3.8$ & $55.4 \pm 1.2$ & $<0.001$ \\
\hline Gender & & & & & $<0.001$ \\
\hline Male & $14(50.0)$ & $15(65.2)$ & $5(83.3)$ & $85(90.4)$ & \\
\hline Female & $14(50.0)$ & $8(34.8)$ & $1(16.7)$ & $9(9.6)$ & \\
\hline
\end{tabular}

Categorical variables are presented as $n$ (\%). HCC, hepatocellular carcinoma; FNH, focal nodular hyperplasia; HCA, hepatocellular adenoma; SD, standard deviation.

TABle 3: Comparison of ADC, $D$, and $K$ values between hepatic benign nodules and the HCC group.

\begin{tabular}{lccc}
\hline & Hepatic benign nodules & HCC & $P$ values \\
\hline $\mathrm{ADC}\left(\times 10^{-3} \mathrm{~mm}^{2} / \mathrm{s}\right)$ & $1.691 \pm 0.067$ & $1.239 \pm 0.039$ & $<0.001^{*}$ \\
$D\left(\times 10^{-3} \mathrm{~mm}^{2} / \mathrm{s}\right)$ & $2.571 \pm 0.116$ & $1.872 \pm 0.596$ & $<0.001^{*}$ \\
$K$ & $0.856 \pm 0.026$ & $1.062 \pm 0.318$ & $<0.001^{*}$ \\
\hline
\end{tabular}

*Mann-Whitney $U$ test. HCC, hepatocellular carcinoma; $K$, kurtosis; $D$, diffusivity; ADC, apparent diffusion coefficient.

TABle 4: $P$ values for intergroup differences using the MannWhitney $U$ test.

\begin{tabular}{lccc}
\hline \multirow{2}{*}{ Groups } & \multicolumn{3}{c}{ Parameters } \\
& $\begin{array}{c}\mathrm{ADC} \\
\left(\times 10^{-3} \mathrm{~mm}^{2} / \mathrm{s}\right)\end{array}$ & $\begin{array}{c}D \\
\left(\times 10^{-3} \mathrm{~mm}^{2} / \mathrm{s}\right)\end{array}$ & $K$ \\
\hline HCC-FNH & $<0.001$ & $<0.001$ & 0.011 \\
HCC-hemangioma & $<0.001$ & $<0.001$ & $<0.001$ \\
FNH-hemangioma & 0.260 & 0.985 & 0.001 \\
HCC-HCA & 1.000 & 1.000 & 1.000 \\
FNH-HCA & 0.085 & 0.127 & 1.000 \\
HCA-hemangioma & 0.046 & 1.000 & 0.086 \\
\hline
\end{tabular}

HCC, hepatocellular carcinoma; FNH, focal nodular hyperplasia; HCA, hepatocellular adenoma; $K$, kurtosis; $D$, diffusivity; ADC, apparent diffusion coefficient.
Taouli and Koh [29] summarized different ADC cutoffs for diagnosing malignant hepatic $\left(1.4-1.6 \times 10^{-3} \mathrm{~mm}^{2} / \mathrm{sec}\right)$ with a sensitivity of $74 \%-100 \%$ and specificity of $77 \%-100 \%$. However, Sutherland et al. [30] and Sandrasegaran et al. [31] found no difference between ADC values of solid benign liver lesions and malignant lesions. One of the main reasons is that $\mathrm{ADC}$ values are related to $b$ values used for image acquisition, while DWI sequences lack standardization. This may also be the result of the different sample sizes or the different methods used to calculate ADC values.

As an advanced DWI model, DKI has been increasingly implemented for providing more precise information of tissue characteristics than conventional DWI. Compared with conventional DWI assuming the Gaussian behavior of water 


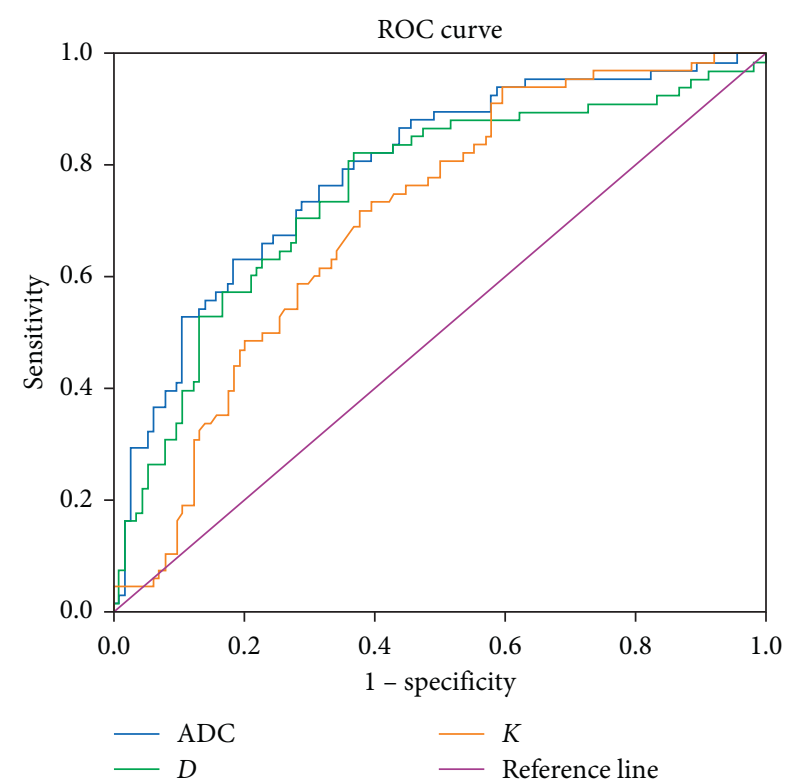

FIgURE 6: ROC curve analysis shows the accuracy of the $K$ value (AUC 0.761 ; sensitivity $65.5 \%$; specificity $79.0 \%$ ), $D$ value (AUC 0.770 ; sensitivity $83.6 \%$; specificity $59.6 \%$ ), and ADC value (AUC 0.826 ; sensitivity $80.6 \%$; specificity $70.8 \%$ ) for differentiating between benign nodules and HCC.

diffusion, DKI could quantify non-Gaussian behavior of water diffusion, which is closer to the movement and distribution of water molecules within biologic tissues. DKI not only provides a corrected apparent diffusion coefficient (ADC) but also reflects the deviation of tissue diffusion from a Gaussian distribution. On the other hand, DKI is better fitted with image signal attenuation than the monoexponential model, and it can reveal the heterogeneity of the lesions more precisely [32]. In hepatic nodules, the incidence of necrosis, hemorrhage, and cystic degeneration is higher in malignant tumors due to the active degree of proliferation, which makes malignant tumor more heterogeneous in radiological appearance than benign tumor. However, some of the hepatic benign nodules may also demonstrate obvious heterogeneous appearance. Examples include central scar, fiber separation, arteriovenous fistula or inflammation in $\mathrm{FNH}$, small thrombus or cyst degeneration in hepatic cavernous hemangioma, and hemorrhage or steatosis in hepatic adenoma $[30,31,33]$. This finding is consistent with the previously published results evaluating the application of DKI on the diagnostic process for other malignancies. For example, Falk Delgado et al. [34] showed that the $K$ value had high diagnostic performance of high- and low-grade glioma because of high heterogeneity in high-grade glioma; In the rectal study, Zhu et al. [35] showed that compared with ADC values, the $K$ value was more valuable in the identification of high and low grades of rectal adenocarcinoma and lymph node metastasis.

There are relatively few studies on liver DKI due to low signal-to-noise ratio (SNR) and image artifacts. Goshima et al. showed that the accuracy of $K$ value assessment of HCC viability was higher than that of the ADC value [36]. Rosenkrantz et al. [37] used DKI in fresh liver explants and found the DKI model may have added value in
HCC characterization in comparison with a standard monoexponential model of DWI [37]. However, our study demonstrated the values of $D$ and $K$ of DKI are not better than the ADC of conventional DWI for preoperative diagnosis of the hepatic nodules. One possible explanation is that $K$ values did not provide stronger correlation with cellularity, it reflected structural heterogeneity among tumors with varying degrees of cellularity, and the main influencing factors are complexity of lesion microstructure. In addition, there is a difference in the heterogeneity between different benign hepatic nodules due to their corresponding unique and complex microstructures [38]. Given the above, the diagnosis efficiency of $K$ values may be limited in hepatic malignancy when compared to its application in other organs. In addition, $D$ values and ADC values of malignant lesions were significantly lower than those of benign lesions, and $D$ values were also shown to have a lower performance than ADC. This can be explained by the fact that the $D$ value is a corrected ADC value related to the Gaussian behavior, which is also influenced by $K$ values $[39,40]$.

Different number of $b$ values is crucial for reliable DKI data fitting. Previous abdominal DKI studies have indicated that DKI sequence of body needs at least three $b$ values (maximum optimal range of $1500 \sim 2000 \mathrm{~s} / \mathrm{mm}^{2}$ ), and $3 \mathrm{di}$ rections is applied to each $b$ value [40]. In our study, DKI was performed with six $b$ values ranging from 0 to $2000 \mathrm{~s} / \mathrm{mm}^{2}$ in three orthogonal directions. Actually, the highest $b$ value at $2000 \mathrm{~s} / \mathrm{mm}^{2}$ in the liver also resulted in lower signal-to-noise ratio (SNR) and faster signal decay of the transverse relaxation [28]. The DKI was acquired with a free breathing sequence allowing greater patient comfort. And, patients must perform respiratory training before MRI examination to minimise breathing artifacts.

In clinical settings, contrast enhancement has been widely used in preoperative qualitative diagnosis of focal lesions in the liver, especially in cases of suspected primary hepatocellular carcinoma. For patients with renal failure and contrast agent contraindications, DWI technology provides a kind of noninvasive examination method that is more conducive to impact on the clinical management [41]. The DKI model, an emerging MRI technology, is more sensitive to tissue microstructural complexity and heterogeneity in high $b$ values. However, as mentioned above, at high $b$ values, it accelerates the T2 relaxation, which, in turn, reduces the signal-to-noise ratio significantly. In addition, it is difficult to obtain good image quality for high $b$ values owing to respiratory motion artifact [42]. All things considered, conventional DWI has a higher significance to be popularized in clinic.

Our study had the following limitations. Firstly, only HCC, HCA, FNH, and hemangioma were enrolled in the study. Other hepatic nodules such as regenerative nodule $(\mathrm{RN})$, dysplastic nodule (DN), intrahepatic cholangiocellular carcinoma, and liver metastases have not been included in this study due to the small sample of cases. Moreover, selective bias could not be avoided in our study as atypical FNH and hemangioma might not have been included in the study. In future studies, we will try to involve more types of hepatic nodules to evaluate 
the efficacy of DKI for distinguishing HCC from other hepatic nodules. Secondly, in image analysis, the ROIs were only delineated in the maximum transection of the lesion, which may result in selection bias. Thirdly, there were two $b$ values less than $200 \mathrm{~s} / \mathrm{mm}^{2}$ in DWI scanning, which did not comply with the optimal value principle of the biexponential model, and the relationship between liver perfusion parameters and hepatic nodules was not statistically analyzed. Further, despite no significant effect of Gd-EOB-DTPA on DWI had been previously reported [27], the effect of Gd-EOB-DTPA on DKI remains unclear. Consequently, further study is required to explore the DKI acquisition delay after Gd-EOB-DTPA administration.

In conclusion, DKI, based on non-Gaussian diffusion reveals the tissue heterogeneity. $D, K$, and $\mathrm{ADC}$ parameters of DKI and conventional DWI have higher efficiency for differentiating between benign and malignant hepatic nodules which are useful in the preoperative diagnosis of hepatic nodules. However, the data from this study did not demonstrate the values of $D$ and $K$ of DKI were better than those of the ADC of conventional DWI in differentiating HCC from benign hepatic nodules.

$\begin{array}{ll}\text { Abbreviations } \\ \text { DKI: } & \text { Diffusion kurtosis imaging } \\ \text { DWI: } & \text { Diffusion-weighted imaging } \\ \text { HCC: } & \text { Hepatocellular carcinoma } \\ \text { FNH: } & \text { Focal nodular hyperplasia } \\ \text { HCA: } & \text { Hepatocellular adenoma } \\ \text { K: } & \text { Kurtosis } \\ \text { D: } & \text { Diffusivity } \\ \text { ADC: } & \text { Apparent diffusion coefficient } \\ \text { ROI: } & \text { Region of interest } \\ \text { AUC: } & \text { Area under the curve } \\ \text { OR: } & \text { Odds ratio } \\ \text { PLC: } & \text { Primary liver cancer } \\ \text { MRI: } & \text { Magnetic resonance imaging } \\ \text { MVI: } & \text { Microvascular invasion } \\ \text { T1WI: } & \text { T1-weighted images } \\ \text { T2WI: } & \text { T2-weighted images } \\ \text { SE-EPI: } & \text { Spin-echo echo planar imaging } \\ \text { SNR: } & \text { Signal-to-noise ratio } \\ \text { HBP: } & \text { Hepatobiliary phase } \\ \text { FA: } & \text { Flip angle } \\ \text { RN: } & \text { Regenerative nodule } \\ \text { DN: } & \text { Dysplastic nodule. } \\ & \end{array}$

\section{Data Availability}

The datasets generated during the current study are not publicly available because of patient privacy but are available from the corresponding author on reasonable request.

\section{Ethical Approval}

The study was approved by the institute review board of the First Affiliated Hospital of Sun Yat-Sen University.

\section{Disclosure}

The Grants-in-Aid supported this study just financially and had no role in the design of the study; collection, analysis, and interpretation of data; and writing the manuscript.

\section{Conflicts of Interest}

The authors declare that they have no conflicts of interest.

\section{Authors' Contributions}

Yingmei Jia and Huasong Cai contributed equally to this work and should be considered as co-first authors. STF and ZPL designed the research and were responsible for quality control of data. YJ, LX, and ZD performed the follow-up survey and collected the data. YX and YL performed the statistical analysis of data. HC and MW performed MRI scan for patients. YJ and STF wrote the manuscript, and all authors edited and made critical revisions to the article. All authors have read and approved the final manuscript.

\section{Acknowledgments}

This work was funded by the National Natural Science Foundation of China (81771908, 81571750, and 81770654).

\section{References}

[1] J. M. Lee, J.-H. Yoon, I. Joo, and H. S. Woo, "Recent advances in CT and MR imaging for evaluation of hepatocellular carcinoma," Liver Cancer, vol. 1, no. 1, pp. 22-40, 2012.

[2] K. A. McGlynn, L. Tsao, A. W. Hsing, S. S. Devesa, and J. F. Fraumeni, "International trends and patterns of primary liver cancer," International Journal of Cancer, vol. 94, no. 2, pp. 290-296, 2001.

[3] W. Chen, R. Zheng, S. Zhang et al., "Cancer incidence and mortality in China, 2013," Cancer Letters, vol. 401, pp. 63-71, 2017.

[4] K. Sartorius, B. Sartorius, C. Aldous, P. S. Govender, and T. E. Madiba, "Global and country underestimation of hepatocellular carcinoma (HCC) in 2012 and its implications," Cancer Epidemiology, vol. 39, no. 3, pp. 284-290, 2015.

[5] W. Chen, R. Zheng, P. D. Baade et al., "Cancer statistics in China, 2015," CA: A Cancer Journal for Clinicians, vol. 66, no. 2, pp. 115-132, 2016.

[6] L. Saba, M. di Martino, C. N. de Cecco, C. Catalano, and M. Piga, "Diagnostic confidence of computed tomography and magnetic resonance in focal liver pathology," European Journal of Gastroenterology \& Hepatology, vol. 27, no. 1, pp. 97-101, 2015.

[7] T. K. Kim, E. Lee, and H.-J. Jang, "Imaging findings of mimickers of hepatocellular carcinoma," Clinical and Molecular Hepatology, vol. 21, no. 4, pp. 326-343, 2015.

[8] J. Ricke, M. Seidensticker, and K. Mohnike, "Noninvasive diagnosis of hepatocellular carcinoma in cirrhotic liver: current guidelines and future prospects for radiological imaging," Liver Cancer, vol. 1, no. 1, pp. 51-58, 2012.

[9] U. Motosugi, P. Bannas, K. Sano, and S. B. Reeder, "Hepatobiliary MR contrast agents in hypovascular hepatocellular carcinoma," Journal of Magnetic Resonance Imaging, vol. 41, no. 2, pp. 251-265, 2015. 
[10] R. Kaewlai and H. Abujudeh, "Nephrogenic systemic fibrosis," American Journal of Roentgenology, vol. 199, no. 1, pp. W17-W23, 2012.

[11] P. Ni, Y. Lin, Q. Zhong, Z. Chen, K. Sandrasegaran, and C. Lin, "Technical advancements and protocol optimization of diffusion-weighted imaging (DWI) in liver," Abdominal Radiology, vol. 41, no. 1, pp. 189-202, 2016.

[12] N. Bharwani and D. M. Koh, "Diffusion-weighted imaging of the liver: an update," Cancer Imaging, vol. 13, no. 2, pp. 171-185, 2013.

[13] S. Shankar, N. Kalra, A. Bhatia et al., "Role of diffusion weighted imaging (DWI) for hepatocellular carcinoma (HCC) detection and its grading on 3T MRI: a prospective study," Journal of Clinical and Experimental Hepatology, vol. 6, no. 4, pp. 303-310, 2016.

[14] V. Granata, R. Fusco, S. Filice et al., "The current role and future prospectives of functional parameters by diffusion weighted imaging in the assessment of histologic grade of HCC," Infectious Agents and Cancer, vol. 13, no. 1, 2018.

[15] J. H. Jensen, J. A. Helpern, A. Ramani, H. Lu, and K. Kaczynski, "Diffusional kurtosis imaging: the quantification of non-gaussian water diffusion by means of magnetic resonance imaging," Magnetic Resonance in Medicine, vol. 53, no. 6, pp. 1432-1440, 2005.

[16] J. H. Jensen and J. A. Helpern, "MRI quantification of nongaussian water diffusion by kurtosis analysis," NMR in Biomedicine, vol. 23, no. 7, pp. 698-710, 2010.

[17] L. Mannelli, S. Kim, C. H. Hajdu, J. S. Babb, T. W. I. Clark, and B. Taouli, "Assessment of tumor necrosis of hepatocellular carcinoma after chemoembolization: diffusion-weighted and contrast-enhanced MRI with histopathologic correlation of the explanted liver," American Journal of Roentgenology, vol. 193, no. 4, pp. 1044-1052, 2009.

[18] I. R. Kamel, E. Liapi, D. K. Reyes, M. Zahurak, D. A. Bluemke, and J.-F. H. Geschwind, "Unresectable hepatocellular carcinoma: serial early vascular and cellular changes after transarterial chemoembolization as detected with MR imaging," Radiology, vol. 250, no. 2, pp. 466-473, 2009.

[19] P. Raab, E. Hattingen, K. Franz, F. E. Zanella, and H. Lanfermann, "Cerebral gliomas: diffusional kurtosis imaging analysis of microstructural differences," Radiology, vol. 254 , no. 3 , pp. $876-881,2010$.

[20] X. Wang, N. Tu, T. Qin, F. Xing, P. Wang, and G. Wu, "Diffusion kurtosis imaging combined with DWI at 3-T MRI for detection and assessment of aggressiveness of prostate cancer," American Journal of Roentgenology, vol. 211, no. 4, pp. 797-804, 2018.

[21] Y. Dai, Q. Yao, G. Wu et al., "Characterization of clear cell renal cell carcinoma with diffusion kurtosis imaging: correlation between diffusion kurtosis parameters and tumor cellularity," NMR in Biomedicine, vol. 29, no. 7, pp. 873-881, 2016.

[22] K. Sun, X. Chen, W. Chai et al., "Breast cancer: diffusion kurtosis MR imaging-diagnostic accuracy and correlation with clinical-pathologic factors," Radiology, vol. 277, no. 1, pp. 46-55, 2015.

[23] H. Wang, X.-Y. Wang, X.-X. Jiang, and Z.-X. Ye, "Comparison of diffusion-weighted with T2-weighted imaging for detection of small hepatocellular carcinoma in cirrhosis," Academic Radiology, vol. 17, no. 2, pp. 239-243, 2010.

[24] J. Budjan, E. A. Sauter, F. G. Zoellner et al., "Diffusion kurtosis imaging of the liver at 3 tesla: in vivo comparison to standard diffusion-weighted imaging," Acta Radiologica, vol. 59, no. 1, pp. 18-25, 2018.
[25] F. Agnello, M. Ronot, D. C. Valla, R. Sinkus, B. E. Van Beers, and V. Vilgrain, "High- $b$-value diffusion-weighted MR imaging of benign hepatocellular lesions: quantitative and qualitative analysis," Radiology, vol. 262, no. 2, pp. 511-519, 2012.

[26] T. Tamada, K. Ito, A. Yamamoto et al., "Hepatic hemangiomas: evaluation of enhancement patterns at dynamic MRI with gadoxetate disodium," American Journal of Roentgenology, vol. 196, no. 4, pp. 824-830, 2011.

[27] K. Saito, Y. Araki, J. Park et al., "Effect of Gd-EOB-DTPA on T2-weighted and diffusion-weighted images for the diagnosis of hepatocellular carcinoma," Journal of Magnetic Resonance Imaging, vol. 32, no. 1, pp. 229-234, 2010.

[28] N. Galea, V. Cantisani, and B. Taouli, "Liver lesion detection and characterization: role of diffusion-weighted imaging," Journal of Magnetic Resonance Imaging, vol. 37, no. 6, pp. 1260-1276, 2013.

[29] B. Taouli and D.-M. Koh, "Diffusion-weighted MR imaging of the liver," Radiology, vol. 254, no. 1, pp. 47-66, 2010.

[30] T. Sutherland, E. Steele, F. van Tonder, and K. Yap, "Solid focal liver lesion characterisation with apparent diffusion coefficient ratios," Journal of Medical Imaging and Radiation Oncology, vol. 58, no. 1, pp. 32-37, 2014.

[31] K. Sandrasegaran, F. M. Akisik, C. Lin, B. Tahir, J. Rajan, and A. M. Aisen, "The value of diffusion-weighted imaging in characterizing focal liver masses," Academic Radiology, vol. 16, no. 10, pp. 1208-1214, 2009.

[32] Y. Bai, Y. Lin, J. Tian et al., "Grading of gliomas by using monoexponential, biexponential, and stretched exponential diffusion-weighted MR imaging and diffusion kurtosis MR imaging," Radiology, vol. 278, no. 2, pp. 496-504, 2016.

[33] A. Muhi, T. Ichikawa, U. Motosugi et al., "High- $b$-value diffusion-weighted MR imaging of hepatocellular lesions: estimation of grade of malignancy of hepatocellular carcinoma," Journal of Magnetic Resonance Imaging, vol. 30, no. 5, pp. 1005-1011, 2009.

[34] A. Falk Delgado, M. Nilsson, D. van Westen, and A. Falk Delgado, "Glioma grade discrimination with MR diffusion kurtosis imaging: a meta-analysis of diagnostic accuracy," Radiology, vol. 287, no. 1, pp. 119-127, 2018.

[35] L. Zhu, Z. Pan, Q. Ma et al., "Diffusion kurtosis imaging study of rectal adenocarcinoma associated with histopathologic prognostic factors: preliminary findings," Radiology, vol. 284, no. 1, pp. 66-76, 2017.

[36] S. Goshima, M. Kanematsu, Y. Noda, H. Kondo, H. Watanabe, and K. T. Bae, "Diffusion kurtosis imaging to assess response to treatment in hypervascular hepatocellular carcinoma," American Journal of Roentgenology, vol. 204, no. 5, pp. W543-W549, 2015.

[37] A. B. Rosenkrantz, E. E. Sigmund, A. Winnick et al., "Assessment of hepatocellular carcinoma using apparent diffusion coefficient and diffusion kurtosis indices: preliminary experience in fresh liver explants," Magnetic Resonance Imaging, vol. 30, no. 10, pp. 1534-1540, 2012.

[38] S. Ahlawat and L. M. Fayad, "Diffusion weighted imaging demystified: the technique and potential clinical applications for soft tissue imaging," Skeletal Radiology, vol. 47, no. 3, pp. 313-328, 2018.

[39] L. Filli, M. Wurnig, D. Nanz, R. Luechinger, D. Kenkel, and A. Boss, "Whole-body diffusion kurtosis imaging," Investigative Radiology, vol. 49, no. 12, pp. 773-778, 2014.

[40] A. B. Rosenkrantz, A. R. Padhani, T. L. Chenevert et al., "Body diffusion kurtosis imaging: basic principles, applications, and considerations for clinical practice," Journal of Magnetic Resonance Imaging, vol. 42, no. 5, pp. 1190-1202, 2015. 
[41] O. Tokgoz, E. Unlu, I. Unal et al., "Diagnostic value of diffusion weighted MRI and ADC in differential diagnosis of cavernous hemangioma of the liver," African Health Sciences, vol. 16, no. 1, pp. 227-233, 2016.

[42] V. Vandecaveye, F. De Keyzer, C. Verslype et al., "Diffusionweighted MRI provides additional value to conventional dynamic contrast-enhanced MRI for detection of hepatocellular carcinoma," European Radiology, vol. 19, no. 10, pp. 2456-2466, 2009. 


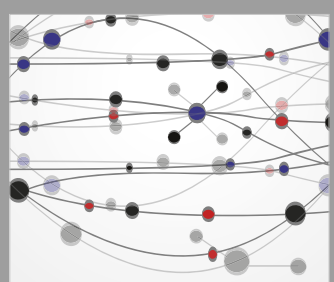

The Scientific World Journal
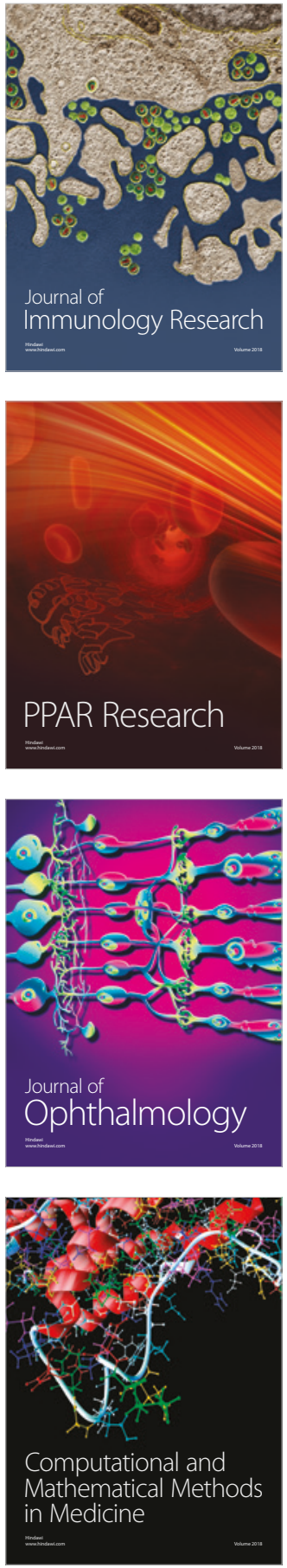

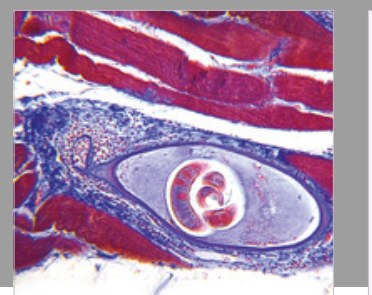

Gastroenterology Research and Practice

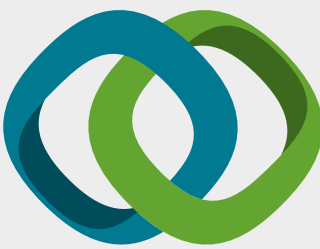

\section{Hindawi}

Submit your manuscripts at

www.hindawi.com
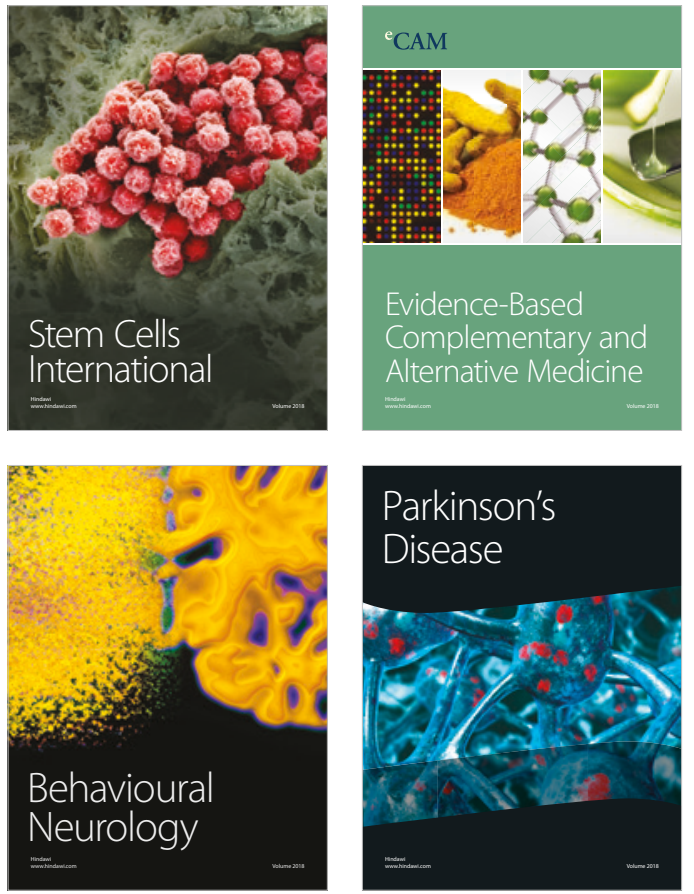

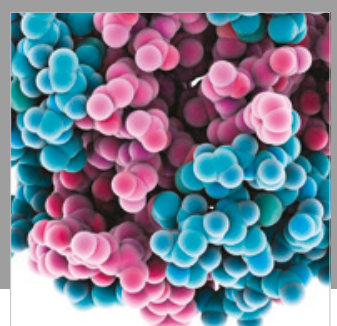

ournal of

Diabetes Research

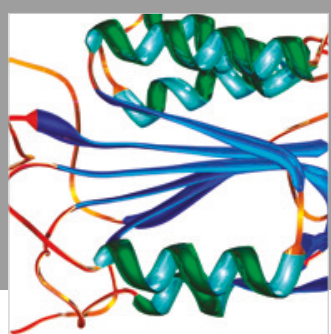

Disease Markers
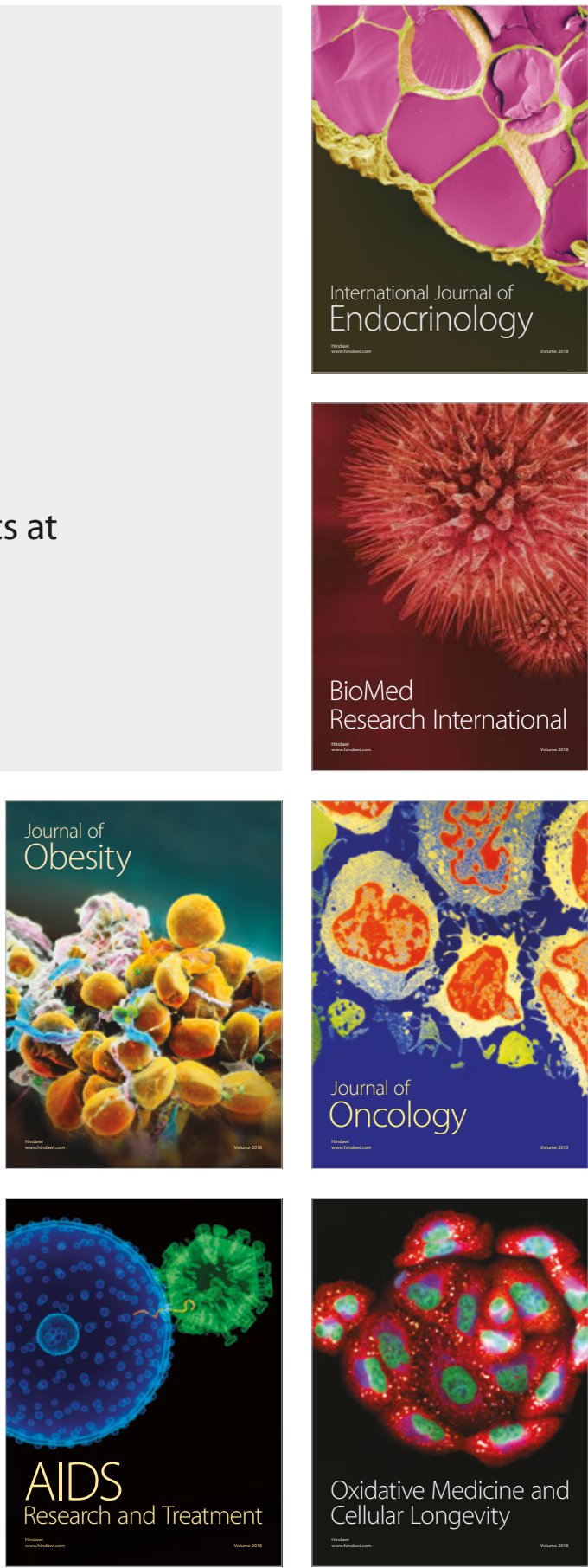